\title{
Spatial Analysis of Esophageal Cancer Mortality in a High-risk Population in China: Consistent Clustering Pattern in 1970-74 and 2011-13
}

\author{
Kou Kou', Xiaolei Guo², Peter Baade ${ }^{3}$, Zilong Lu², Zhentao Fu², Jie Chu², \\ Aiqiang $\mathrm{Xu}^{2}$, Jiandong Sun ${ }^{1 *}$
}

\begin{abstract}
Purpose: Almost half of the global esophageal cancer (EC) deaths occurred in China. This study aims to examine the geographic spread of EC mortality in two periods in a large Chinese population. Methods: Age-standardized mortality rates (ASMRs) for 140 county-level units in Shandong Province during the periods 1970-74 and 2011-13 were derived using data from the First National Cause-of-Death Survey and the Shandong Death Registration System, respectively. ASMRs were smoothed using Area-to-Area Poisson kriging technique. Spatial scan statistics were used to detect spatial clusters with higher EC mortality and clusters with greater temporal changes in EC mortality. Results: The provincial average ASMR decreased from 13.0 per 100,000 in 1970-74 to 5.8 in 2010-13. Almost all counties or districts have experienced a decrease in EC mortality, while the reduction was particularly pronounced in the mid-west region. This study has identified a geographical cluster with much higher EC mortality rates and the clustering pattern has largely unchanged over the past 40 years. Residents living in the cluster during 1970-74 were 2.7 (95\% CI: 2.2-3.4) times more likely to die from EC than the rest of the province. The corresponding risk ratio for the 2011-13 cluster was 3.7 (95\% CI: 2.8-5.0). Conclusions: This study detected a geographically defined subpopulation in Shandong, China with much higher risk of dying from EC. This spatial pattern has been consistent over the past few decades. The results suggest the key drives for geographic variations in esophageal cancer may not have changed.
\end{abstract}

Keywords: Esophageal cancer- mortality- spatial pattern- temporal change- China

Asian Pac J Cancer Prev, 19 (11), 3161-3166

\section{Introduction}

Esophageal cancer (EC) is an important public health issue in China. It is the fourth leading cause of cancer-related death, causing 197,800 deaths nationwide in 2013 (Zhou et al., 2016), accounting for almost half of the global deaths due to EC (Fitzmaurice et al., 2017).

Squamous cell carcinoma (SCC) is the predominant histological type of EC in China (Pennathur et al., 2013). Definitive risk factors for SCC mortality in the Chinese population are unclear, but likely to include tobacco smoking, alcohol drinking, consumption of high-temperature beverages, poverty, and environmental pollution (Zhang et al., 2014; Chen et al., 2015; Liang et al., 2017). The economic boom during the 1980s and 1990s in China has been accompanied by environmental degradation, including a severe deterioration in the water quality of the country's rivers and lakes. An increasing number of studies have raised the concern that the dumping of untreated wastewater in densely populated areas may contribute to the high EC mortality rate in China (Zhang et al., 2003; Ebenstein, 2012; Zhang et al., 2014).

Spatial analysis is an important tool in health research, allowing the identification of spatial patterns and clusters that often stimulate research to establish causative relationships. International studies conducted in different geographic locations have identified regional clusters with higher EC mortality (Aragonés et al., 2007; Drahos et al., 2013). However, since most of the studies are cross-sectional studies, it is unclear whether the observed spatial patterns have changed over time. The temporal changes in spatial patterns are important to identify potential risk factors, especially for the environmental and social ones which vary with time and geographic locations. For example, if water pollution is an influential contributor to EC mortality, we would expect to see a mortality increase in areas with water quality deterioration.

Shandong Province in China has a higher EC mortality rate when compared with the Chinese average 
(Zhou et al., 2016). This paper aims to detect the changes in EC mortality across space and time in Shandong. The study will answer two questions: i) What is the current geographic spread of esophageal cancer mortality? ii) How did the spatial pattern change over time?

\section{Materials and Methods}

\section{Study site}

Shandong Province is a coastal province on the lower reaches of the Yellow River in North-East China. The province covers an area of 157,100 square kilometres. It is the second most populous province in China, with nearly 98.5 million people, of which $99 \%$ belong to the Han ethnic group. Shandong is made up of 17 prefecture-level regions administrating 140 county-level units (Shandong Statistical Yearbook Editorial Committee, 2015).

\section{Data sources}

EC age-standardized mortality rates (ASMRs) data for 1970-74 were extracted from the First National Cause-of-Death Survey (Xu et al., 1979). The survey in Shandong covered all geographic areas with a representative sample of $33.3 \%$ of the total population. Specifically, the survey was conducted in 122 county-level units. Of these, 18 units conducted a census of the whole population; while 104 units surveyed a representative sample of $20 \%$ of the whole population. This representiveness was tested by taking a $20 \%$ random sample from two of the 18 census county-level units, and finding that the estimated mortality rates were similar to that of the census. Causes of death was derived through a mix of medical certification and 'verbal autopsy (VA)' procedures, applied according to standard guidelines by a team of physicians (Yang et al., 2005a). VA is a primary source of information about causes of death in countries, like China, where the majority of deaths occur at home. Study has shown that VA methods perform reasonably well in identifying deaths from EC with the sensitivity as $96.1 \%$ and specificity as 99.7\% in Chinese population (Yang et al., 2005b).

The 2011-13 EC-specific death data were collected from the Shandong Death Registration System (SDRS). SDRS collected death data through local hospitals and community health service stations based on a national standard protocol (National Health and Family Planning Commission of the People's Republic of China, 2014). Briefly, all the residents died in Shandong Province have death certificates generated by registered physicians. Cause-of-death coding is based on the International Classification of Disease, 10th Revision (ICD-10) and was double checked by physicians' direct managers within seven days after death registration. The quality control for the SDRS data has been described in previous publication (Fu et al., 2017).

In this study, deaths caused by a malignant neoplasm of the esophagus $(\mathrm{C} 15)$ according to the ICD-10, in the population residing in Shandong Province during 1st January 2011 and 31st December 2013 were extracted from the SDRS data base. The extracted EC death dataset presents the numbers of EC deaths in each of the 140 county-level units divided by gender and age group.
The 2011-13 population data for each county-level unit by gender and the age group were obtained from Shandong CDC, who generated their estimates based on data from local police departments and statistical bureaus.

\section{Statistical methods}

Only ASMR estimates already age-standardised to the 1964 Chinese population were available for the 1970-74 period. Therefore, we also standardised the 2011-13 mortality rates of the 140 county-level units to the 1964 Chinese population. Both the 1970-74 and 2011-13 ASMR Shandong maps were created using R software (Version 3.3.3, Bell Laboratories).

To minimise the noise caused by unreliable extreme rates computed from sparsely populated geographical units and for areas with a low frequency of EC death, both 2011-13 and 1970-74 ASMRs of the 140 county-level units were smoothed using Area-to-Area (ATA) Poisson kriging technique. The Poisson kriging technique has been described in detail previously (Goovaerts, 2006) and was implemented using the public-domain executable poisson-kriging.exe (Goovaerts, 2005).

The spatial scan statistic (Kulldorff, 1997) was used to detect local clusters with higher EC mortality rates (the smoothed ASMRs). Briefly, the spatial scan statistic uses a very large number of overlapping circles with different sizes and in different locations. Each circle contains a different set of neighbouring geographic units. The likelihood of each circle being a cluster is calculated, with the final cluster being the one with the maximum likelihood over all circles evaluated. The spatial scan statistic was calculated using SaTScan (v9.4.4, National Cancer Institute, USA). The 95\% confidence intervals ( $95 \%$ CIs) for the relative risk of identified clusters were calculated manually according to the $\mathrm{p}$ value (Altman and Bland, 2011). Considering the ATA Poisson kriging technique borrows information from neighbouring areas to smooth mortality rates, this may increase the likelihood of detecting a cluster. Therefore, we also calculated the spatial scan statistic on crude ASMRs to test this potential.

Differences in ASMRs between 2011-13 and 1970-74 were calculated for the 140 units. The spatial scan statistic was also used to detect clusters with greater changes of ASMRs between the two periods and test their statistical significance.

\section{Results}

During 1970-74, a total of 14,966 EC deaths were documented out of $81,295,782$ person-years, resulting a crude mortality rate of 18.41 per 100,000 person-years. In 2011-13, 45,737 EC deaths were reported out of $285,823,471$ person-years, with a crude mortality rate of 15.98 per 100,000 person-years.

\section{Age-standardized mortality rates}

In 1970-74, the overall ASMR (by 1964 Chinese population) of EC in Shandong Province was estimated as 13.0 per 100,000 person-years. The rate decreased considerably to 5.8 in $2010-2013$. Males had higher 
Table 1. Mortality Rate (1/105) of Esophageal Cancer in 2011-13 and 1970-74 in Shandong Province

\begin{tabular}{lccc}
\hline Mortality rates & Gender & $1970-74(95 \% \mathrm{CI})$ & $2011-13(95 \% \mathrm{CI})$ \\
\hline Crude rate & Both & $18.4(18.1-18.7)$ & $16.0(15.9-16.2)$ \\
& Male & $25.8(25.4-26.3)$ & $23.8(23.5-24.1)$ \\
& Female & $10.9(10.6-11.3)$ & $8.0(7.8-8.1)$ \\
M/F ratio & 2.4 & 3.0 \\
ASMR* & Both & $13.0(12.8-13.2)$ & $5.8(5.7-5.8)$ \\
& Male & $18.2(17.9-18.6)$ & $9.3(9.2-9.4)$ \\
& Female & $7.7(7.5-8.0)$ & $2.4(2.3-2.4)$ \\
\hline
\end{tabular}

*ASMR, age-standardized mortality rate by 1964 Chinese standard population; M/F ratio, the ratio of male rate to female rate

Table 2. Results of the Spatial Scan Statistics of Esophageal Cancer Age-adjusted Mortality Risk in Shandong Province, 1970-74 and 2011-13

\begin{tabular}{|c|c|c|c|c|}
\hline Year & Cluster radius $(\mathrm{km})$ & Population* $(100,000)$ & Relative risk $(95 \% \mathrm{CI})$ & p-value \\
\hline \multicolumn{5}{|l|}{ Persons } \\
\hline 1970-74 & 40.1 & 95 & $2.7(2.2-3.4)$ & $<0.001$ \\
\hline 2011-13 & 39.2 & 71 & $3.7(2.8-5.0)$ & $<0.001$ \\
\hline \multicolumn{5}{|l|}{ Males } \\
\hline $1970-74$ & 26.1 & 18 & $3.7(2.8-4.8)$ & $<0.001$ \\
\hline 2011-13 & 39.2 & 36 & $3.5(2.6-4.5)$ & $<0.001$ \\
\hline \multicolumn{5}{|l|}{ Females } \\
\hline $1970-74$ & 39.2 & 37 & $3.3(2.5-4.2)$ & $<0.001$ \\
\hline $2011-13$ & 49.4 & 71 & $4.2(3.0-5.6)$ & $<0.001$ \\
\hline
\end{tabular}

*The population of the clusters are 2011-13 average population by gender.

EC mortality than females. The male to female ratio increased from 2.4 in 1970-74 to 4.0 in 2011-13 (Table 1). On the county level, in 1970-74 the ASMRs ranged widely between 2.1 and 60.5 per 100,000 person-years, and between 0.4 and 29.1 per 100,000 person-years in 2011-13. The mortality rates for EC have decreased in $93.6 \%(n=131)$ of the county-level units in Shandong between the two periods (Figure 1).

\section{Spatial patterns}

The darker shading of the maps (Figure 2) showing the ASMRs for the 140 county-level units for 1970-74 compared to 2011-13 highlight the comprehensive decrease in EC mortality across Shandong. However, the spatial pattern remained very similar; those areas with higher ASMRs in 1970-74 were virtually identical to the units observed in 2011-13 (Figure 2A). Smoothing the rates using the ATA Poisson kriging approach made little difference to the overall spatial pattern within Shandong for both time periods (Figure 2B).

\section{Clusters of mortality rate}

The spatial scan statistic identified a significant clustering of areas having higher EC mortality in both time

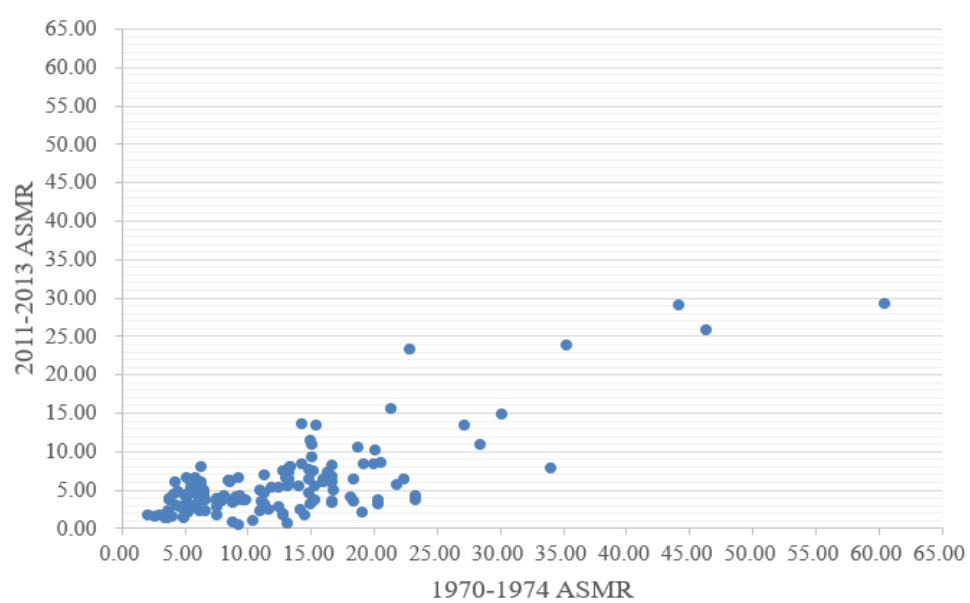

Figure 1. Age-standardized Mortality Rates of Esophageal Cancer in 140 County-level Units, 1970-74 and 2011-13, Shandong, China. Each dot represents a pair of age-standardized mortality rates per 100,000 person-years in a countylevel unit in Shandong Province in 1970-74 (x value) and 2011-13 (y value). 
A

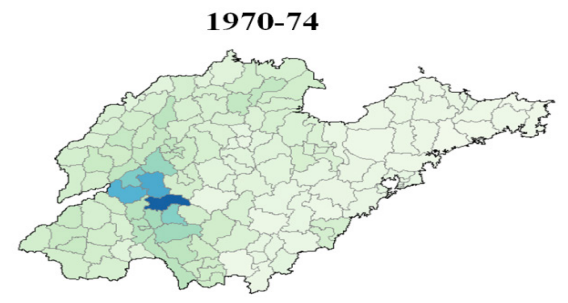

B

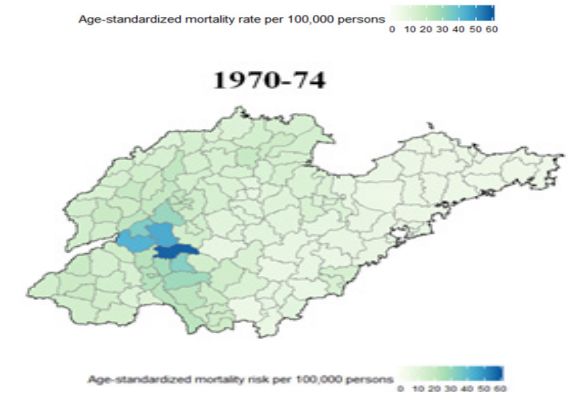

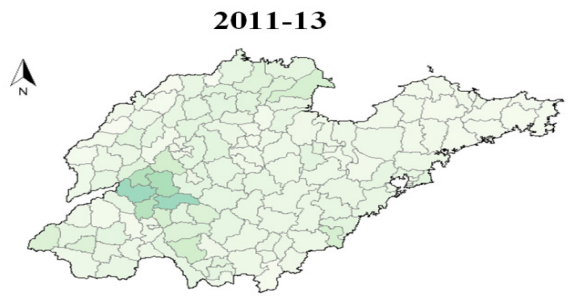

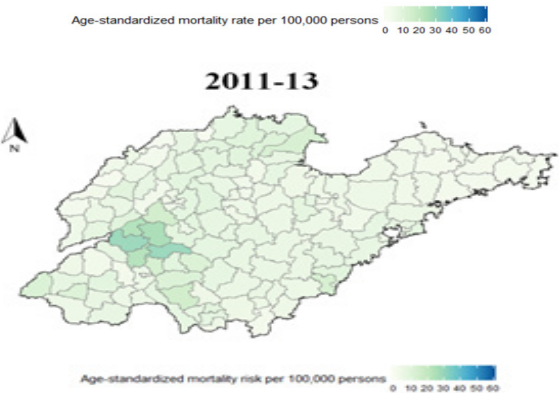

Figure 2. A, Age- standardized Mortality rates of 140 County-level Units in Shandong, 1970-74 and 2011-13. B, Smoothed Age-standardized Mortality Rates of 140 County-level Units in Shandong Using Poisson Kriging, 1970-74 and 2011-13.

periods and for males, females, and persons. For persons, residents living in the $1970-74$ cluster were nearly three times more likely to die from EC than the Shandong average (mortality risk ratio $=2.7,95 \% \mathrm{CI}$ : 2.2-3.4). The corresponding estimate for the geographical cluster identified in the 2011-13 data was 3.7 (95\% CI: 2.8-5.0) (Table 2).

Given the geographical definition of the EC mortality cluster changed between the years, direct comparison of the relative risks was not possible. However, there was substantial overlap between the clusters identified; of the 15 country-level units included in either cluster, 8 were included in both (Figure 3). The cluster identified using 2011-13 cohort contained ten county-level units, with 7.1 million residents, accounting for $7.4 \%$ of the combined Shandong population. Among them, $70.0 \%$ of the population are rural residents, which is higher than the Shandong average proportion (64.2\%).

The spatial scan statistic results from the crude ASMRs were consistent with the results calculated using smoothed ASMRs (results not shown).

\section{Clusters of mortality change}

There was evidence that the reduction in mortality
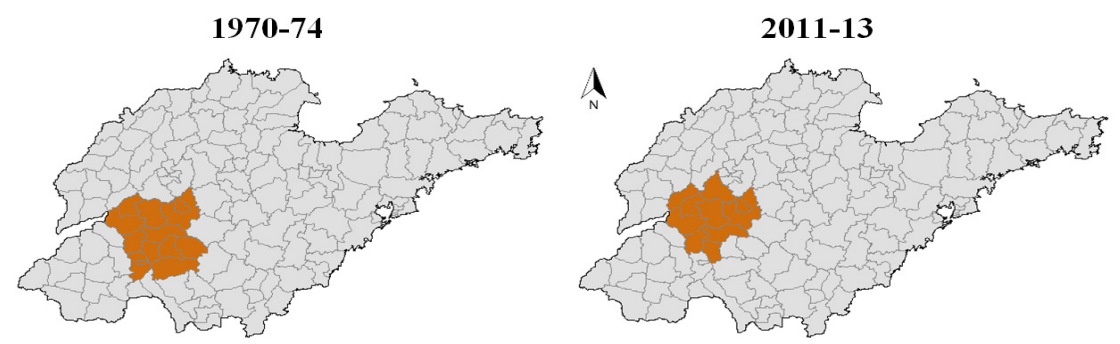

Figure 3. Clusters Identified by Spatial Scan Statistic (SaTScan) with Higher Age-standardized Mortality Rate (Smoothed) in Shandong Province, 1970-74 and 2011-13

(A)

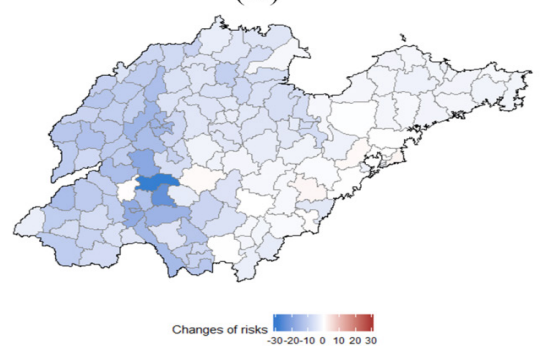

(B)

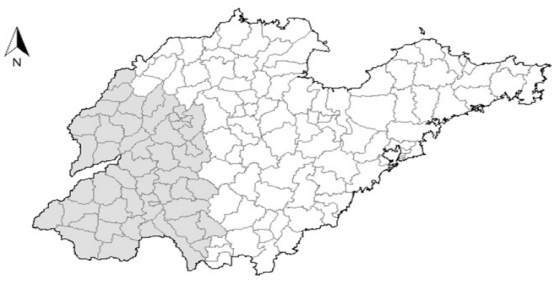

Figure 4. Changes of Age-standardized Mortality Rates (Smoothed) between 1970-74 and 2011-13 and Region with Larger Changes of Age-standardized Mortality rate in Shandong Province 
rates between the two periods was unevenly spread over the province (Figure $4 \mathrm{~A}$ ). The spatial scan statistic identified a region with greater reduction of mortality risks in the mid-west part of Shandong (Figure 4 B). The large-decrease region in Figure $4 \mathrm{~B}$ includes the identified high-mortality clusters in Figure 3.

\section{Discussion}

This study identified a high-mortality risk cluster of EC in Shandong. The location of this cluster has not changed much after forty years. The temporal consistency of the high-mortality cluster in the mid-west region of Shandong suggests that the primary contributors to this geographical clustering have been stable during the study period. Case-control studies concluded that alcohol and tobacco use, consumption of hot beverages and foods, low socio-economic status (SES), and water pollution are playing important roles in the development of EC in Asian countries (Islami et al., 2009; Zhang et al., 2014; Chen et al., 2015). Considering EC is a highly fatal disease, with an overall 5-year relative survival rate less than $25 \%$, the above-mentioned factors increasing EC incidence generally increase its mortality (Rustgi and El-Serag, 2014).

Tobacco and alcohol consumption has been reported as a risk factor for $\mathrm{EC}$ in Chinese population (Yu et al., 2018). However, from the existing evidence, it is difficult to conclude that tobacco and alcohol consumption alone is associated with the consistently higher EC mortality in the cluster area. The prevalence of smoking in Shandong has been reported to be higher than the national average (Guo et al., 2004), while very few studies have identified spatial varication of tobacco and alcohol use in Shandong. Jiangsu Province borders Shandong in the north and, like Shandong, has above-average EC mortality rates and large geographic variation (Zhou et al., 2016). A study conducted in two locations with different EC mortality rates in Jiangsu reported that the prevalence of alcohol and tobacco use is similar in both high- and low-mortality areas (Wu et al., 2006).

It is possible that the intake of burning-hot food and beverages could contribute to the higher mortality in the cluster area in Shandong. A recent population-based cohort study conducted in a Chinese population suggested that high-temperature food combined with either alcohol or tobacco consumption was associated with a greater risk (risk ratio: 5.0 vs 2.5) for EC than tobacco and alcohol consumption alone (Yu et al., 2018). Anecdotally, residents living in the mid-west region of Shandong (Tai'an City) eat a variant of corn porridge at burning temperatures for almost every meal. Tai'an is a prefecture-level city of which five of its six county-level units are located in the high-mortality cluster in both time periods.

Low SES could partially explain the higher mortality rate in the cluster area. International studies in high-risk areas reported that higher EC risk was related to lower income, lower education level, and a higher percentage of employment in agriculture (Mohebbi et al., 2011). When compared to the Shandong average, the 2011-13 high-mortality cluster has a slightly higher proportion of rural residents (70\% vs 64\%) which usually have low SES. Moreover, a report showed that the 2008 per capita consumption expenditure of rural residents living in the 2011-13 high-mortality cluster is below Shandong's average (Li and Haibao, 2011).

Water pollution has attracted attention in epidemiological studies of EC in China in recent years, with some Chinese studies finding an association between the quality of water and the risk of EC (Zhang et al., 2003; Zhang et al., 2014). Given the high-mortality EC cluster is located at the junction of Yellow River and Dawen River, the rapid deterioration of their water quality since the 1990s, due to contaminants from industrial and agricultural activities (Wang et al., 2015) needs to be considered in light of the results of this study. In particular, there has been only minimal change in the location of the high EC mortality cluster despite the deterioration in river water quality, and the EC mortality rate has decreased, rather than the increase that would be expected if the deterioration of water quality in those rivers impacted on EC mortality.

The decrease in mortality rate in Shandong over the last 40 years is consistent with national and international studies (Semnani et al., 2006; Chen et al., 2016). However, the mortality decrease is not evenly spread geographically. We found evidence of a cluster located in the mid-west region of Shandong with significantly greater decrease of ASMRs. Interestingly, this large-decrease region overlaps the high-mortality cluster identified in this study. The reasons behind this phenomenon are unclear. Our hypothesis is that since the health professionals and the public in the high-mortality areas tend to have a higher level of awareness of this disease, the patients there will have a better chance to get early detection and treatment, which could reduce the mortality rate. A study revealed that $99 \%$ of the rural residents living in a city of the high-mortality cluster (Feicheng) were aware of EC and the value of cancer screening (Yang, 2009).

\section{Limitations}

The data collection methods differ between the two periods. The 1970-74 mortality data were collected by a survey and the 2011-13 data were collected using a population registration system. Although for both periods, diagnosis methods are mainly based on histopathology, radiology, or clinical symptoms, these methods and hospital records may have improved over time. It is likely that the mortality rates in the 1970s were underestimated because of lower efficacy in diagnosis and case reporting. Therefore, the decrease of EC mortality rates may have been even larger over forty years. However, since the data collection methods remain the same within each period, the identified spatial patterns have not been influenced by this limitation.

In addition, our analyses were based on the total EC mortality with no information on histological and pathological types. EC mainly consists of two different histological types (SCC and adenocarcinoma) with different epidemiologic features. Although research data have shown that more than $90 \%$ of EC cases in China are SCC (Liang et al., 2017), a small amount of patient 
could be dead of adenocarcinoma. Since we do not have relevant information in our data, we did not differentiate our analysis by the histological type.

In Conclusion, our comprehensive analysis demonstrates significant regional variations in the mortality of EC in Shandong Province and identified a stable high-mortality cluster that existed over 40 years in a large, racially homogeneous population. The results could be helpful to guide surveillance and control activities of EC. Examining the spatial patterns is a preliminary step towards further explorations that might identify environmental or behavioural risk factors. We hypothesize here that the high-mortality cluster observed may be influenced by regional exposure to factors such as specific dietary habits and low SES. Further studies that can test these hypotheses are needed to get a better understanding of the aetiology of EC in high-risk areas.

\section{Acknowledgements}

The research was supported by Queensland University of Technology and the "Shandong Science and Technology Development Plan Project (2016GSF201231)". We would also like to show our appreciation to Dr. Susanna Cramb from Cancer Council Queensland for her valuable comments on an earlier version of the manuscript.

\section{References}

Altman DG, Bland JM (2011). How to obtain the confidence interval from a $\mathrm{P}$ value. $B M J, \mathbf{3 4 3}, \mathrm{d} 2090$.

Aragonés N, Ramis R, Pollán M, et al (2007). Oesophageal cancer mortality in Spain: a spatial analysis. BMC Cancer, 7,3 .

Chen W, Zheng R, Baade PD, et al (2016). Cancer statistics in China, 2015. CA Cancer J Clin, 66, 115-32.

Chen Y, Tong Y, Yang C, et al (2015). Consumption of hot beverages and foods and the risk of esophageal cancer: a meta-analysis of observational studies. BMC Cancer, 15, 449.

Drahos J, Wu M, Anderson WF, et al (2013). Regional variations in esophageal cancer rates by census region in the United States, 1999-2008. PLoS One, 8, e67913.

Ebenstein A (2012). The consequences of industrialization: evidence from water pollution and digestive cancers in China. Rev Econ Stat, 94, 186-201.

Fitzmaurice C, Allen C, Barber RM, et al (2017). Global, regional, and national cancer incidence, mortality, years of life lost, years lived with disability, and disability-adjusted life-years for 32 cancer groups, 1990 to 2015: a systematic analysis for the global burden of disease study. JAMA Oncol, 3, 524-48.

Fu Z, Li Y, Lu Z, et al (2017). Lung cancer mortality clusters in Shandong Province, China: how do they change over 40 years?. Oncotarget, 8, 88770.

Goovaerts P (2005). Geostatistical analysis of disease data: estimation of cancer mortality risk from empirical frequencies using Poisson kriging. Int J Health Geogr, 4, 31.

Goovaerts P (2006). Geostatistical analysis of disease data: accounting for spatial support and population density in the isopleth mapping of cancer mortality risk using area-to-point Poisson kriging. Int J Health Geogr, 5, 52.

Guo X, Ma J, Li W, et al (2004). Cross-sectional survey on smoking among rural and urban residents in Shandong
Province (in Chinese). J Inform Prev Med, 10, 13-5.

Islami F, Kamangar F, Nasrollahzadeh D, et al (2009). Socio-economic status and oesophageal cancer: results from a population-based case-control study in a high-risk area. Int J Epidemiol, 38, 978-88.

Kulldorff M (1997). A spatial scan statistic. Commun Stat Theory Methods, 26, 1481-96.

Li S-n, Haibao W (2011). Analysis on status and countermeasures of the wealth gaps in rural areas of Shandong Province (in Chinese). J Anhui Agric, 39, 603-5.

Liang H, Fan J-H, Qiao Y-L (2017). Epidemiology, etiology, and prevention of esophageal squamous cell carcinoma in China. Cancer Biol Med, 14, 33-41.

Mohebbi M, Wolfe R, Jolley D, et al (2011). The spatial distribution of esophageal and gastric cancer in Caspian region of Iran: An ecological analysis of diet and socio-economic influences. Int J Health Geogr, 10, 13.

Pennathur A, Gibson MK, Jobe BA, et al (2013). Oesophageal carcinoma. Lancet, 381, 400-12.

Rustgi AK, El-Serag HB (2014). Esophageal carcinoma. NEngl J Med, 371, 2499-509.

Semnani S, Sadjadi A, Fahimi S, et al (2006). Declining incidence of esophageal cancer in the Turkmen Plain, eastern part of the Caspian Littoral of Iran: a retrospective cancer surveillance. Cancer Detect Prev, 30, 14-9.

Wang Y, Yang L, Kong L, et al (2015). Spatial distribution, ecological risk assessment and source identification for heavy metals in surface sediments from Dongping Lake, Shandong, East China. Catena, 125, 200-5.

Wu M, Zhao J-K, Hu X-S, et al (2006). Association of smoking, alcohol drinking and dietary factors with esophageal cancer in high-and low-risk areas of Jiangsu Province, China. World $J$ Gastroenterol, 12, 1686.

Xu H, Xu D, Zhang X, et al (1979). The investigation of causeof-death for malignant tumor in Shandong Province (in Chinese). Introdoction, Shandong Department of Cancer Control and Prevention, pp 1-4.

Yang G, Hu J, Rao KQ, et al (2005a). Mortality registration and surveillance in China: history, current situation and challenges. Popul Health Metr, 3,3 .

Yang G, Rao C, Ma J, et al (2005b). Validation of verbal autopsy procedures for adult deaths in China. Int J Epidemiol, 35, 741-8.

Yu C, Tang H, Guo Y, et al (2018). Effect of hot tea consumption and its interactions with alcohol and tobacco use on the risk for esophageal cancer: A population-based cohort study. Ann Intern Med, 168, 489-7.

Zhang X-L, Bing Z, Xing Z, et al (2003). Research and control of well water pollution in high esophageal cancer areas. World J Gastroenterol, 9, 1187-90.

Zhang X, Zhuang D, Ma X, et al (2014). Esophageal cancer spatial and correlation analyses: Water pollution, mortality rates, and safe buffer distances in China. J Geogr Sci, 24, 46-58.

Zhou M, Wang H, Zhu J, et al (2016). Cause-specific mortality for 240 causes in China during 1990-2013: a systematic subnational analysis for the Global Burden of Disease Study 2013. Lancet, 387, 251-72.

This work is licensed under a Creative Commons AttributionNon Commercial 4.0 International License. 